\title{
Birmingham Intervention Tent Technique (BITT): A Technical Note
}

\author{
R. Rajakulasingam ${ }^{1} \quad$ J. Kho ${ }^{1}$ \\ G. Almeer ${ }^{1}$ \\ C. Azzopardi ${ }^{1}$ \\ S. L. James ${ }^{1}$ \\ R. Botchu ${ }^{1}$
}

1 Department of Musculoskeletal Imaging, Royal Orthopaedic Hospital, Birmingham, UK

Indian J Radiol Imaging 2021;31:521-523.

\begin{abstract}
Address for correspondence Rajesh Botchu, FRCR, Department of Musculoskeletal Radiology, The Royal Orthopedic Hospital, Bristol Road South, Northfield, Birmingham, UK, (e-mail: drbrajesh@yahoo.com).
\end{abstract}
Abstract
Keywords
- intervention
- tent
- technique

Objective We describe a novel and safe CT biopsy technique that we have termed the "Birmingham intervention tent technique (BITT)." This technique is ideal for biopsying osseous lesions where a direct approach is not possible due to difficult positioning. Methods The BITT uses a plastic surgical forceps clamp attached at an angle to the biopsy needle, creating a tent shape. The finger rings of the forceps is stabilized on the table.

Results In our institution, we have already used the BITT successfully in over 10 cases. Conclusion The BITT is an inexpensive and reproducible technique.

\section{Introduction}

Several techniques have recently been described in the literature to aid image-guided biopsy of osseous lesions. ${ }^{1-3}$ All of them rely on a relatively even or flat skin surface to ensure needle stability. In cases where there is uneven skin surface/dip in skin contour, a conventional biopsy approach can be challenging. This is also true for parts of the body where prominent angulation is needed, for example, in the posterior neck (cervical spine) or in cortical and periosteal lesions where there is paucity of overlying soft tissue. We describe an innovative, inexpensive, and simple technique of stabilizing and manipulating the biopsy needle in the described situations. This has been termed the "Birmingham intervention tent technique (BITT)."

\section{Technique}

BITT uses the surgical plastic forceps clamp, which is readily available in most operating theater dressing packs. The clamp is applied directly to the biopsy needle. Depending on the angle required, the clamp can be attached anywhere along the needle until stability is achieved. The finger rings of the forceps are rested on the table or similar structure. The angle made between the biopsy needle and clamp takes the shape

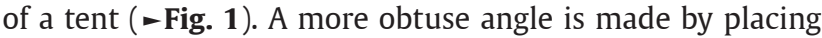
the clamp close to the needle tip; conversely, a sharper angle is made by placing the clamp closer to the proximal end of the biopsy needle. The clamp finger rings provide enough stability; in other words, no fixation is needed for the patient. Furthermore, the weight of the applied clamp and two finger rings is enough to counteract the weight of the biopsy needle, again ensuring stability. If the needle needs to be advanced further, the clamp can be removed, the needle repositioned, and clamp reapplied until it is firmly anchored in the bone. Informed consent were obtained from patients.

\section{Discussion}

In the authors' experience, BITT is an effective method for biopsying lesions where a direct approach is not possible due to an uneven skin surface or difficult anatomic access. published online

July 27,2021
DOI https://doi.org/

$10.1055 / \mathrm{s}-0041-1733467$ ISSN 0971-3026 (c) 2021. Indian Radiological Association.

This is an open access article published by Thieme under the terms of the Creative Commons Attribution-NonDerivative-NonCommercial-License, permitting copying and reproduction so long as the original work is given appropriate credit. Contents may not be used for commercial purposes, or adapted, remixed, transformed or built upon. (https://creativecommons.org/licenses/by-nc-nd/4.0/). Thieme Medical and Scientific Publishers Private Ltd. A-12, Second Floor, Sector -2, NOIDA -201301, India 


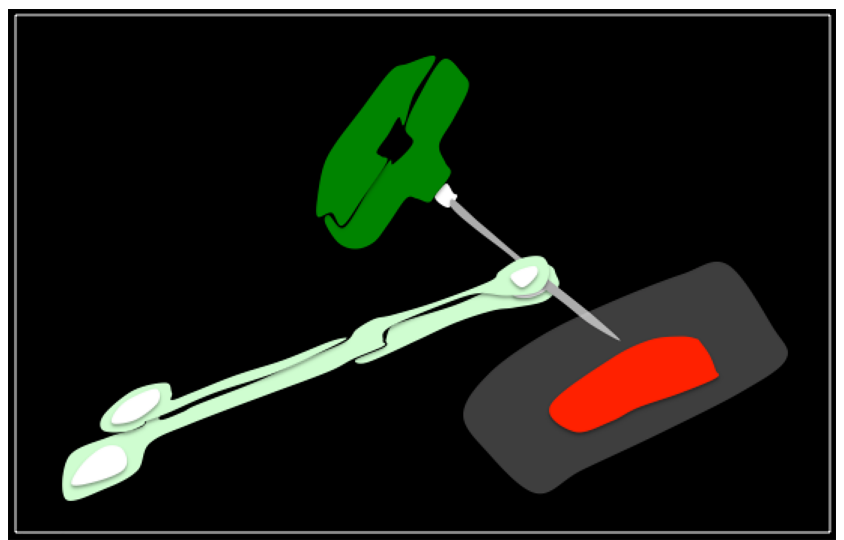

Fig. 1 Illustration of the "XXXX intervention tent technique (BITT)." The biopsy needle is held with a plastics forceps clamp, which is not attached to the drape. The weight of the clamp alone is enough to support the weight of the biopsy needle.

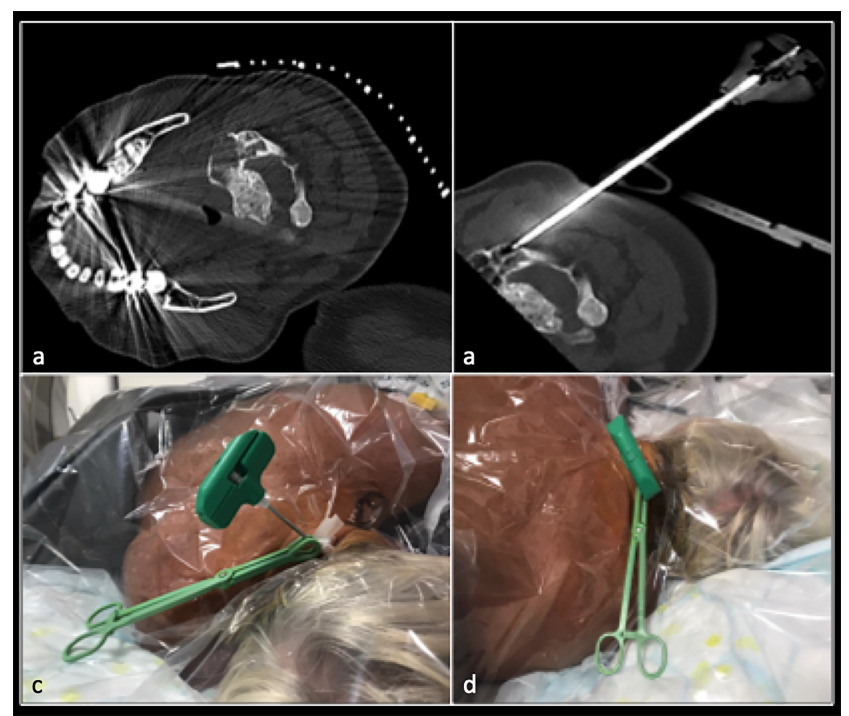

Fig. 2 The lesion within the left lateral aspect of the $C 2$ vertebral body was to be biopsied. As the shoulder was in the way for a direct approach, the clamp could be applied to the needle and biopsied using an angulated entry point ( $\mathbf{A}$ and $\mathbf{B})$. The images ( $\mathbf{C}$ and $\mathbf{D})$ show how the clamp is positioned to the side of the patient, avoiding difficult maneuvering over the shoulder/upper back.

It can also be used in lesions where an angulated approach, not directly perpendicular to the CT beam, is needed. In such cases, using the biopsy needle alone in an angulated manner with no accessory stabilizing force may result in instability and the needle being displaced.

-Fig. 2 demonstrates the described technique. The lesion for biopsy was within the lateral aspect of the C2 vertebral body. The natural hump of the shoulder and upper back meant that the "SAHNA" technique was not possible, and presence of hair made use of the "dual steristrip" technique difficult. To tackle this, an angulated approach was used by attaching forceps clamp to the biopsy needle. The clamp allowed an angle to be maintained without the needle falling out.

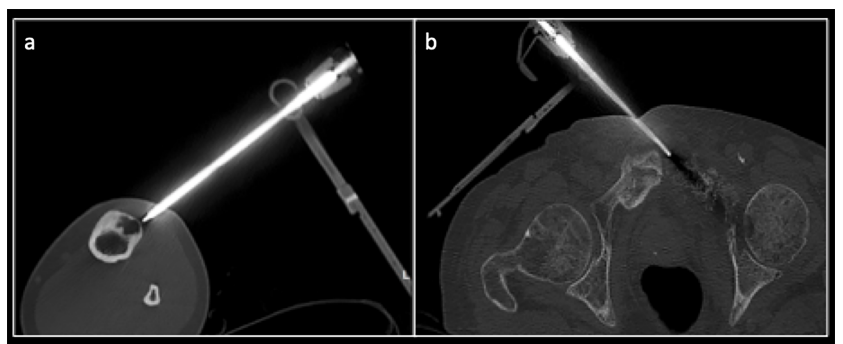

Fig. 3 Lesions within the anterior tibia (A) and superior pubic ramus (B) biopsied using the "XXXXX intervention tent technique (BITT)." The correct biopsy plane and trajectory can be easily planned and maintained by applying a clamp to the biopsy needle.

BITT can also be used in other CT biopsy procedures. It can act as a guide to ensure correct trajectory is maintained. It is especially helpful in cases where an angulated entry point is required. - Fig. 3 illustrates the technique being used in biopsying lesions within the tibia and superior pubic ramus. When the practitioner advances the needle, the clamp position aids the radiologist to maintain the correct direction and angle. This is useful for osseous lesions, where a direct approach perpendicular to the scanning plane is not feasible. The technique is also helpful for bones where an angled approach may be used, for example, the clavicle or pubic rami which have a natural anatomical angular inclination. This should avoid angling the needle too inferior or superior, possibly missing the correct part of the lesion. The clamp is also ideal for lesions where there is abundant soft tissue, requiring a longer needle length, such as the posterior neck seen in (-Fig. 2). Attaching the clamp as demonstrated is enough to balance even longer length needles.

In most institutions, radiologists may place a small needle tip in the skin to decide a trajectory after local anesthetic is administered. With a clamp, the biopsy needle tract can be optimized after skin entry, and then modified and maintained throughout the procedure.

Currently, there are only three published techniques highlighting methods for stabilizing CT biopsy needles. In the "dual steristrip" technique devised by McLoughlin and authors, one steristrip is placed on the biopsy needle, proximal to the entry site, and the other at roughly 90 degrees on the skin surface, away from needle entry point. ${ }^{1}$ However, in cases where an angulated entry point is required, more steristrips are needed for stabilization. There is always a risk that the steristrips will not hold if stretched, leading to needle instability. Nabi and coauthors have recommended using an inverted gallipot with a central hole to stabilize the biopsy needle. ${ }^{2}$ The hole is made via a bone drill and then placed over the skin entry site. The needle is then advanced through the hole and into the lesion. However, angling or repositioning the needle may prove to be difficult, given the narrow hole aperture. With a wider entry point, the angle may be too obtuse for the needle to fit through the hole at the required position.

The "SAHNA" technique uses a mepore dressing, stuck down to the clamp roughly halfway up its length. ${ }^{3}$ This is ideal 
for balancing the needle in superficial osseous lesions where not much bony purchase exists. However, it again may not be suitable for cases requiring an angulated approach. Sticking the clamp down may even alter the angle of entry slightly; the SAHNA technique is mainly recommended for superficial osseous lesions perpendicular to the scanning plane.

Commercially available needle holders such as Seestar (Apriomed) and Simplify (NeoRad AS) have been developed. ${ }^{4}$ These are external devices which attach around the biopsy needle, forming a construct to help in effective planning. However, they tend to increase biopsy time and there is equivocal evidence as to whether they reduce hand radiation when compared with a free hand technique. ${ }^{5}$ It would also be very laborious to detach and reapply the device if multiple needle adjustments are needed. The lack of extra radiation dose and ease of manipulation in real-time allows the BITT to be used even in the most technically challenging situations.

Conflict of Interest

The authors declare no conflict of interest

\section{References}

1 McLoughlin E, Iqbal A, Patel A, James SL, Botchu R. Dual steristrip technique: a novel use of steristrips to reduce operator radiation dose during CT-guided intervention. Skeletal Radiol 2019;48(10):1617-1620

2 Nabi M, Teh J. The use of plastic gallipots for balancing needles in CT-guided procedures. Clin Radiol 2019;74(7):570. e19-570.e20

3 Rajakulasingam R, Iqbal A, James SL, Botchu R. Surface adhesive and hand-aided needle-assisted biopsy technique (SAHNA. Skeletal Radiol 2019; (e-pub ahead of print). doi: 10.1007/s00256-019-03320-y

4 Kroes MW, Busser WMH, Fütterer JJ, et al. Assessment of needle guidance devices for their potential to reduce fluoroscopy time and operator hand dose during C-arm cone-beam computed tomography-guided needle interventions. J Vasc Interv Radiol 2013;24(6):901-906

5 Sarmento S, Pereira JS, Sousa MJ, et al. The use of needle holders in CTF guided biopsies as a dose reduction tool. J Appl Clin Med Phys 2018;19(1):250-258 